\title{
VARIABILIDADE GENÉTICA MTHFR NO DESENVOLVIMENTO DA DOENÇA ARTERIAL CORONÁRIA
}

\author{
Patrícia Matos Biselli', Alexandre Rodrigues Guerzoni², Eny Maria Goloni-Bertollo*3, Moacir Fernandes de Godoy ${ }^{4}$, Juliana Aparecida \\ Barcelos Abou-Chahla ${ }^{5}$, Érika Cristina Pavarino-Bertelli ${ }^{6}$ \\ Trabalho realizado na Unidade de Pesquisa em Genética e Biologia Molecular - UPGEM, Faculdade de Medicina de São José do Rio Preto - FAMERP
}

\author{
*Correspondência: \\ Unidade de Pesquisa \\ em Genética e Biologia \\ Molecular - UPGEM \\ Departamento de Biologia \\ Molecular \\ Faculdade de Medicina de \\ São José do Rio Preto - \\ FAMERP \\ Av. Brigadeiro Faria Lima, \\ n. ${ }^{\circ} 5416$ - Bloco U-6 \\ São José do Rio Preto - SP \\ CEP: 15.090-000 \\ Telefone: (17) 3201-5720 \\ Fax: (17) 3201-5708 \\ eny.goloni@famerp.br
}

\begin{abstract}
RESUMO
OвJEтIvo. Concentração elevada de homocisteína (Hcy) é considerada um fator de risco para doença arterial coronária (DAC). Alterações genéticas da enzima metilenotetrahidrofolato redutase (MTHFR), envolvida no metabolismo da Hcy, podem reduzir sua termolabilidade contribuindo para o desenvolvimento de lesões ateroscleróticas. O objetivo deste estudo foi investigar a relação entre os polimorfismos MTHFR C677T e A1298C e a presença, extensão e gravidade da DAC.

MÉTodos. Foram avaliados 175 pacientes com DAC, confirmada por angiografia e 108 indivíduos sem DAC (grupo controle). O polimorfismo MTHFR C677T foi investigado por reação em cadeia da polimerase (PCR) seguida de digestão enzimática. A genotipagem do polimorfismo MTHFR A1298C foi realizada pela técnica de PCR alelo-específica.

Resultados. A frequência do alelo alterado MTHFR 677 C foi de 0,38 no grupo DAC e 0,37 no grupo controle. Em relação ao alelo polimórfico MTHFR 1298C, a frequência foi de 0,22 e 0,27, respectivamente. As distribuições genotípicas MTHFR C677T e A1298C não diferiram em relação ao número de artérias lesadas $(P>0,05)$. Também não foi observada relação entre o polimorfismo para MTHFR C677T e grau de obstrução arterial coronária $(P>0,05)$, assim como MTHFR A1298C $(P>0,05)$. ConcLusÃo. Nossos resultados não demonstraram associação entre os polimorfismos MTHFR A1298C e MTHFR C677T e presença, extensão ou gravidade da DAC.
\end{abstract}

UnITERmos: Doença arterial coronária. Polimorfismos genéticos. Enzima MTHFR.

\section{INTRODUÇÃO}

Concentração elevada de homocisteína (Hcy) plasmática é considerada um fator de risco para doença arterial coronária $(D A C)^{1}$. Diversas enzimas e cofatores desempenham papel importante no metabolismo desse aminoácido. A enzima 5,10 metilenotetrahidrofolato redutase (MTHFR) catalisa a conversão de 5,10-metilenotetrahifrofolato em 5-metiltetrahidrofolato, principal forma circulante de folato, que é requerido para uma série de vias metabólicas, incluindo a metilação da Hcy para metionina ${ }^{2}$.

$\mathrm{Na}$ espécie humana, concentrações elevadas de Hcy estão associadas à presença do polimorfismo C677T no gene MTHFR, que resulta em termolabilidade e atividade reduzida da enzima MTHFR ${ }^{3}$. Outro polimorfismo no gene MTHFR, uma substituição de adenina para citosina no nucleotídeo 1298, causa mudança de ácido glutâmico para alanina na proteína produzida. A contribuição desse polimorfismo para a atividade enzimática é questionável. Estudo de expressão in vitro da enzima MTHFR mostrou uma redução de $32 \%$ na atividade da enzima mutante 1298 e de $59 \%$ para aquela mutada para ambos os polimorfismos (C677T e A1298C) ${ }^{4}$. Foi observado que a presença do genótipo MTHFR 677CT/1298AA leva à redução de cerca de $30 \%$ da atividade enzimática; enquanto os genótipos homozigoto MTHFR 677CC/1298CC e heterozigoto MTHFR 677CT/1298AC resultam na diminuição desta atividade em 35 a $43 \%$ e de 38 a $50 \%$, respectivamente ${ }^{5}$. Por outro lado, Yamada et al., $(2001)^{6}$ não observaram alteração na função catalítica ou regulatória da enzima na presença da variante MTHFR 1298CC.

Ambos os polimorfismos do gene MTHFR foram considerados fatores de risco para $D A C^{7,8,9,10}$. A associação do polimorfismo MTHFR C677T com DAC foi confirmada por alguns estudos, nos quais a prevalência de portadores do alelo alterado foi maior no grupo DAC em relação aos controles ${ }^{10-14}$ por outro lado, outros trabalhos não confirmam tal associação ${ }^{15,16,17}$. Em relação ao

1. Pós-graduanda em Ciências da Saúde, Faculdade de Medicina de São José do Rio Preto - FAMERP, São José do Rio Preto, SP

2. Doutor pela Faculdade de Medicina de São José do Rio Preto - FAMERP, São José do Rio Preto, SP

3. Professora Adjunta livre-docente do Departamento de Biologia Molecular, Faculdade de Medicina de São José do Rio Preto - FAMERP, São José do Rio Preto, SP

4. Professor Adjunto do Departamento de Cardiologia e Cirurgia Cardiovascular, Faculdade de Medicina de São José do Rio Preto - FAMERP, São José do Rio Preto, SP

5. Graduanda curso de Medicina da Faculdade de Medicina de São José do Rio Preto - FAMERP, São José do Rio Preto, SP

6. Professora Adjunta livre-docente do Departamento de Biologia Molecular, Faculdade de Medicina de São José do Rio Preto - FAMERP, São José do Rio Preto, SP 


\begin{tabular}{lccc}
\hline \multicolumn{3}{c}{$\begin{array}{c}\text { Tabela } 1 \text { - Distribuição das frequências genotípicas dos } \\
\text { polimorfismos MTHFR C677T e A1298C }\end{array}$} \\
\hline Genótipos & DAC & $\begin{array}{c}\text { Controle } \\
\text { (N = 108) }\end{array}$ & Valor de P \\
\hline MTHFR C677T & & & \\
CC, n (\%) & $62(35)$ & $39(36)$ & \\
CT, n (\%) & $93(54)$ & $59(55)$ & 0,847 \\
TT, n (\%) & $20(11)$ & $10(9)$ & \\
MTHFR A1298C & & & \\
AA, n (\%) & $101(58)$ & $54(50)$ & 0,448 \\
AC, n (\%) & $67(38)$ & $49(45)$ & \\
CC, n (\%) & $7(4)$ & $5(5)$ & \\
\hline
\end{tabular}

DAC - Doença arterial coronária

polimorfismo MTHFR A1298C, o alelo alterado foi associado com início precoce da DAC, independente dos níveis de Hcy9 .

O objetivo deste estudo foi investigar a relação entre os polimorfismos C677T e A1298C e presença, extensão e gravidade da DAC.

\section{Métodos}

O grupo de estudo foi composto por 283 indivíduos, caucasóides, atendidos no Hospital de Base de São José do Rio Preto, e submetidos à angiografia coronária. $O$ estudo foi aprovado pela Comissão Nacional de Ética em Pesquisa (CONEP). Os indivíduos foram divididos em dois grupos: grupo DAC, 175 pacientes com obstrução coronária $(60,7 \pm 12,1$ anos; 112 homens e 63 mulheres), e grupo controle, 108 indivíduos sem sinal de lesão aterosclerótica (57,8 8 12,3 anos; 58 homens e 50 mulheres). O diagnóstico de DAC foi confirmado ou excluído por angiografia coronária, analisada por dois observadores em análise cega quantitativa. A extensão da DAC foi determinada de acordo com o número de vasos coronários envolvidos ( 1 a 3), e a gravidade pelo grau de obstrução arterial (estenose <50\%, 50-75\%, > 75-95\%, e $>95 \%$ ). Indivíduos submetidos à cirurgia de revascularização cardíaca, substituição de válvulas cardíacas ou colocação de prótese laminar coronariana foram excluídos do estudo. Embora exista no Brasil vasta miscigenação, foram considerados caucasóides os indivíduos que não apresentaram ascendência de outros grupos étnicos nas três gerações antecedentes ${ }^{18}$.

Amostras de sangue periférico foram coletadas após consentimento livre e esclarecido. DNA genômico foi extraído de leucócitos, de acordo com a técnica descrita por Abdel-Rahman et al. $(1994)^{19}$. A análise do polimorfismo MTHFR C677T foi realizada por reação em cadeia da polimerase (PCR) seguida de digestão enzimática, de acordo com Bova et al. (1999) ${ }^{13}$. Os fragmentos de DNA foram separados por eletroforese em gel de poliacrilamida $9,6 \%$ e submetidos à diferença de potencial de $200 \mathrm{~V}$ por 4 horas. Após a eletroforese, o gel foi corado com nitrato de prata.

O polimorfismo MTHFR A1298C foi investigado pela técnica de PCR alelo-específica segundo Ranjith et al., (2003) ${ }^{20}$, com modificações. Os primers utilizados foram: (Alelo $A$ ) sense $5^{\prime}$ GGA GCT GAC CAG TGA AGA -3' e antissense 5' - TGT GAC CAT TCC GGT TTG -3'; (Alelo C) sense 5' - CTT TGG GGA GCT GAA GGA -3' e anti-sense 5' - AAG ACT TCA AAG ACA CTT G $-3^{\prime}$; (controle positivo de amplificação gênica) sense $5^{\prime}$ - TGA
AGG AGA AGG TGT CTG CGG GA -3' e antissense 5' - AGG ACG GTG CGG TGA GAG TG -3'. A amplificação foi realizada com desnaturação inicial de 2 minutos a $94^{\circ} \mathrm{C}$, seguida de 30 ciclos de 30 segundos a $94^{\circ} \mathrm{C}, 30$ segundos a $58^{\circ} \mathrm{C}$ e 50 segundos a $72^{\circ} \mathrm{C}$. O produto da amplificação foi separado por eletroforese em gel de poliacrilamida a $6 \%$, submetido à diferença de potencial de $200 \mathrm{~V}$ por duas horas e corado com nitrato de prata.

Comparação entre os grupos para distribuições alélicas e genotípicas foi realizada utilizando o Teste Exato de Fisher ou Qui quadrado. O Equilíbrio de Hardy-Weinberg (HWE) foi testado pelo Teste Qui quadrado. Associação entre genótipos e número de artérias obstruídas ou grau de obstrução arterial foi avaliada por Análise de Dependência. As análises estatísticas foram realizadas utilizando o programa Minitab for Windows.

\section{Resultados}

A distribuição genotípica para o polimorfismo MTHFR C677T não diferiu significantemente entre os grupos $(P=0,847)$ (Tabela 1). O genótipo MTHFR 677CT foi prevalente em ambos os grupos ( $54 \%$ nos pacientes com DAC e $55 \%$ nos controles). O alelo polimórfico MTHFR 677T apresentou frequência de 0,38 no grupo DAC e 0,37 no grupo controles, não havendo diferença estatística significante $(P=0,789)$. A distribuição genotípica se mostrou em HWE em ambos os grupos DAC e controle $\left(x_{1}{ }_{1}=2,86 ; P=0,091\right.$ e $x^{2}{ }_{1}=3,40, P=0,065$, respectivamente).

A distribuição genotípica não diferiu em relação ao número de artérias lesadas $(P=0,156)$ (Tabela 2$)$. Também não foi observada relação entre o polimorfismo MTHFR C677T e grau de obstrução arterial coronária $(p=0,993)$ (Tabela 3).

Não houve diferença entre os grupos estudados em relação às distribuições genotípicas $(P=0,448)$ e alélicas $(P=0,271)$ (Tabela 1) para o polimorfismo MTHFR A1298C. O genótipo homozigoto selvagem MTHFR 1298AA foi prevalente em ambos os grupos ( $58 \%$ nos pacientes e $50 \%$ nos controles). A frequência do alelo alterado MTHFR $1298 \mathrm{C}$ foi de 0,22 no grupo DAC e 0,27 no grupo controles. O cálculo para o teste do HWE mostrou que a distribuição genotípica foi semelhante à esperada em ambos os grupos DAC e controles $\left(\mathrm{x}^{2}{ }_{1}=1,01 ; \mathrm{P}=0,313 \mathrm{e}\right.$ $\mathrm{x}_{1}^{2}=2,19, \mathrm{P}=0,138$, respectivamente).

Nenhuma associação foi observada em relação ao número de artérias lesadas ( $P=0,380$ ) (Tabela 2$)$ e grau de obstrução arterial $(P=0,800)$ (Tabela 3$)$.

As frequências dos genótipos dos polimorfismos MTHFR C677T e MTHFR A1298C combinados não diferiram entre pacientes e controles $(P=0,172)$. Também não foi observada associação dos genótipos combinados e número de artérias $(P=0,657)$ ou grau de obstrução coronária $(P=0,350)$.

\section{Dıscussão}

A contribuição das variantes genéticas MTHFR no desenvolvimento de lesões ateroscleróticas se baseia no papel da enzima MTHFR no metabolismo da homocisteína (Hcy). Os mecanismos fisiopatológicos referentes ao papel pró-aterogênico da hiper-homocisteinemia (Hcy > 15 umol/L) ainda não são totalmente esclarecidos. Evidências sugerem que níveis elevados de Hcy induzem a disfunção e lesão endotelial, 
Tabela 2 - Distribuição genotípica dos polimorfismos MTHFR C677T e A1298C em relação ao número de artérias obstruídas

\begin{tabular}{lccc}
\hline & \multicolumn{2}{c}{ Número de artérias obstruídas } \\
\cline { 2 - 4 } Genótipos & Uma artéria & Duas artérias & Três artérias \\
\hline MTHFR C677T & $28(45)$ & $19(30)$ & $15(31)$ \\
CC, $n(\%)$ & $26(41)$ & $37(58)$ & $30(63)$ \\
CT, $n(\%)$ & $9(14)$ & $8(12)$ & $3(6)$ \\
TT, $n(\%)$ & & & $27(56)$ \\
MTHFR A1298C & $33(52)$ & $41(64)$ & $18(38)$ \\
AA, $n(\%)$ & $29(46)$ & $20(31)$ & $3(6)$ \\
AC, $n(\%)$ & $1(2)$ & $3(5)$ & 0,156 \\
CC, $n(\%)$ & & & 0,380 \\
\hline
\end{tabular}

Tabela 3 - Comprometimento coronariano em relação aos polimorfismos MTHFR C677T e A1298C

\begin{tabular}{|c|c|c|c|c|c|}
\hline \multirow[b]{2}{*}{ Genótipos } & \multicolumn{4}{|c|}{ Graus de obstrução arterial } & \multirow[b]{2}{*}{ Valor de $P$} \\
\hline & $<50 \%$ & $50 \%-75 \%$ & $>75 \%-95 \%$ & $>95 \%$ & \\
\hline \multicolumn{6}{|c|}{$\overline{\text { MTHFR C677T }}$} \\
\hline $\mathrm{CC}, n(\%)$ & $6(35)$ & $13(36)$ & $29(35)$ & $14(35)$ & \multirow{3}{*}{0,993} \\
\hline $\mathrm{CT}, n(\%)$ & $9(53)$ & $19(53)$ & $45(55)$ & $20(50)$ & \\
\hline $\mathrm{TT}, n(\%)$ & $2(12)$ & $4(11)$ & $8(10)$ & $6(15)$ & \\
\hline \multicolumn{6}{|c|}{ MTHFR A1298C } \\
\hline$A A, n(\%)$ & $11(65)$ & $20(56)$ & $49(60)$ & $21(52)$ & \multirow{3}{*}{0,800} \\
\hline$A C, n(\%)$ & $6(35)$ & $16(44)$ & $30(36)$ & $15(38)$ & \\
\hline $\mathrm{CC}, n(\%)$ & $0(0)$ & $0(0)$ & $3(4)$ & $4(10)$ & \\
\hline
\end{tabular}

proliferação de células musculares lisas, ativação de plaquetas e formação de trombos ${ }^{21,22,23}$, levando à formação de placas ateroscleróticas.

O polimorfismo C677T do gene MTHFR resulta em termolabilidade e atividade enzimática reduzida, e contribui para o aumento dos níveis de $\mathrm{Hcy}^{3}$. A contribuição da variante polimórfica MTHFR 1298CC na redução da função enzimática não é totalmente esclarecida, uma vez que estudos observaram resultados contraditórios ${ }^{5,6}$. Níveis de Hcy de indivíduos heterozigotos MTHFR 1298AC e homozigotos MTHFR 1298CC não diferiram daqueles com genótipo selvagem MTHFR 1298AA, sugerindo que este polimorfismo isolado não afeta significantemente 0 metabolismo da $\mathrm{Hcy}^{4,6}$, mas pode exercer um efeito moderado na presença da variante $\mathrm{C} 677 \mathrm{~T}^{4}$

No presente estudo, os polimorfismos MTHFR C677T e A1298C e seus genótipos combinados não foram associados com a presença, extensão ou gravidade da DAC. A relação entre o polimorfismo MTHFR C677T e a DAC tem sido avaliada por vários estudos. Nossos resultados corroboram com estudos prévios, nos quais nenhuma associação entre os genótipos MTHFR C677T e a DAC foi encontrada ${ }^{24,15}$. Kebert et al. (2006) ${ }^{24}$ observaram $66,2 \%$ de indivíduos portadores do alelo MTHFR $677 \mathrm{C}$ e $33,8 \%$ portadores do alelo MTHFR 677T em população caucasóide com DAC. Estudo em população brasileira mostrou resultado semelhante e sugere que o polimorfismo C677T da MTHFR não é um fator de risco independente para $D A C^{17}$. A frequência do alelo polimórfico MTHFR 677T foi de 0,24 nos indivíduos com DAC. No presente estudo foi observada frequência de 0,38 do alelo alterado.
Por outro lado, o alelo polimórfico MTHFR 677T já foi associado com doenças vasculares, aterosclerose de carótida, doença arterial obstrutiva e infarto do miocárdio ${ }^{10-14}$. Em DAC, o alelo polimórfico foi observado significantemente mais freqüente nos pacientes em relação aos controles ${ }^{10-14,25}$. Em estudo brasileiro, o polimorfismo foi associado à gravidade da doença, mas não apresentou distribuição significantemente diferente entre pacientes com e sem DAC ${ }^{26}$.

A alteração MTHFR A1298C também tem sido investigada em indivíduos com DAC. Estudo de Szczeklik et al. $(2001)^{9}$ mostraram frequência do alelo polimórfico MTHFR $1298 \mathrm{C}$ significantemente maior em indivíduos com DAC em relação aos controles, independente dos níveis de Hcy. Corroborando com nosso resultado, Abu-Amero et al. (2003) ${ }^{27}$ e Kolling et al. (2004) ${ }^{15}$ não encontraram associação entre os genótipos MTHFR A1298C e presença ou extensão da DAC. Este último não observou diferença na distribuição genotípica tanto para o polimorfismo MTHFR A1298C, quanto para o MTHFR C677T em relação ao número de artérias obstruídas. Em trabalho de Gueant-Rodriguez et al. (2005) ${ }^{16}$ os polimorfismos MTHFR A1298C e MTHFR C677T também não foram associados à DAC.

Os genótipos MTHFR A1298C e MTHFR C677T combinados também foram analisados no presente estudo e não apresentaram relação com a DAC, assim como com a extensão ou gravidade da doença. Corroborando com nossos resultados, Meisel

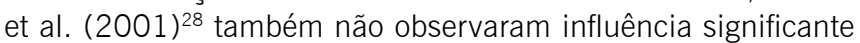
dos polimorfismos MTHFR C677T e A1298C combinados no processo de desenvolvimento da DAC, assim como nos níveis de Hcy. 


\section{Conclusão}

Nossos resultados não demonstraram associação entre os polimorfismos MTHFR A1298C e MTHFR C677T epresença, extensão ou gravidade da DAC. A investigação das concentrações de Hcy e de polimorfismos de outras enzimas envolvidas no metabolismo desse aminoácido poderá contribuir para o esclarecimento da etiologia da DAC.

\section{Agradecimentos}

Agradecemos a colaboração do Prof. Dr. José Antônio Cordeiro na análise estatística e o auxílio financeiro recebido da Fundação de Amparo à Pesquisa do Estado de São Paulo - FAPESP, Coordenação de Aperfeiçoamento de Pessoal de Nível Superior - CAPES e Conselho Nacional de Desenvolvimento Científico e Tecnológico - CNPq.

\section{SUPORTE FinANCEIRO:}

Fundação de Amparo à Pesquisa do Estado de São Paulo - FAPESP (Auxílio Pesquisa), Coordenação de Aperfeiçoamento de Pessoal de Nível Superior - CAPES (Bolsa de Mestrado) e Conselho Nacional de Desenvolvimento Científico e Tecnológico - CNPq (Bolsa de Iniciação Científica).

\section{Conflito de interesse: não há}

\section{SUMMARY}

\section{MTHFR GENETIC VARIABILITY ON CORONARY ARTERY DISEASE DEVELOPMENT}

OBJECTIVE. Increased homocysteine (Hcy) concentration is considered a risk factor for coronary artery disease (CAD). Genetic alterations of the metylenetetrahydrofolate reductase (MTHFR) enzyme could reduce its thermolability and alter the Hcy metabolism, contributing to development of atherosclerotic lesions. Objective of this study was to investigate the relation between MTHFR C677T and A1298C polymorphisms and presence, extension, and severity of CAD.

METHODS. One hundred seventy-five patients with CAD confirmed by angiography, and 108 individuals without CAD (control group) were evaluated. MTHFR C677T polymorphism was investigated by polymerase chain reaction (PCR) followed by enzyme digestion. The genotyping of the MTHFR A1298C polymorphism was performed by PCR allele-specific method.

RESULTS. Frequency of the altered allele MTHFR $677 \mathrm{C}$ was 0.38 in the CAD group and 0.37 in the control group. Regarding the polymorphic allele MTHFR 1298C, frequency was 0.22 and 0.27 , respectively. The genotype distribution MTHFR C677T and A1298C did not differ regarding number of affected vessels $(P>0.05)$. Also, relation between MTHFR C677T polymorphism and degree of arterial obstruction was not observed $(P>0.05)$, as well as the MTHFR A1298C polymorphism $(P>0.05)$.

CONCLUSION. Results did not show association between MTHFR A1298C and MTHFR C677T polymorphisms and presence, extension or severity of CAD. [Rev Assoc Med Bras 2009; 55(3): 274-8]

KEY WORDS: Coronary artery disease. Genetic polymorphisms. MTHFR enzyme.

\section{REFERÊNCIAS}

1. Sadeghian S, Fallahi F, Salarifar M, Davoodi G, Mahmoodian M, Fallah N, et al. Homocysteine, vitamin B12 and folate levels in premature coronary artery disease. BMC Cardiovasc Disord 2006;6:38.

2. Finkelstein JD. The metabolism of homocysteine: pathways and regulation. Eur J Pediatr. 1998;157(Suppl 2):40-4.

3. Haviv YS, Shpichinetsky V, Goldschmidt N, Atta IA, Ben-Yehuda A, Friedman G. The common mutations C677T and A1298C in the human methylenetetrahydrofolate reductase gene are associated with hyperhomocysteinemia and cardiovascular disease in hemodialysis patients. Nephron. 2002;92:120-6.

4. Weisberg IS, Jacques PF, Selhub J, Bostom AG, Chen Z, Ellison RC, et al. The 1298 A3C polymorphism in methylenetetrahydrofolate reductase (MTHFR): in vitro expression and association with homocysteine. Atherosclerosis. 2001;156:409-15.

5. Weisberg I, Tran P, Christensen B, Sibani S, Rozen R. A second genetic polymorphism in methylenetetrahydrofolate reductase (MTHFR) associated with decreased enzyme activity. Mol Genet Metab. 1998;64:169-72.

6. Yamada K, Chen Z, Rozen R, Matthews RG. Effects of common polymorphisms on the properties of recombinant human methylenetetrahydrofolate reductase. Proc Natl Acad Sci USA. 2001;98:14853-8.

7. Mager A, Lalezari S, Shohat T, Birnbaum Y, Adler Y, Magal N, et al. Methylenetetrahydrofolate reductase genotypes and early-onset coronary artery disease. Circulation. 1999;100:2406-10.

8. Payne DA, Chamoun AJ, Seifert SI, Stouffer GA. MTHFR677C >T mutation: a predictor of early-onset coronary artery disease. Thromb Res. 2001;103:275-9.

9. Szczeklik A, Sanak M, Jankowski M, Dropinski J, Czachor R, Musial J, et al. Mutation A1298C of methylenetetrahydrofolate reductase: risk for early coronary disease not associated with hyperhomocysteinemia. Am J Med Genet. 2001:101:36-9.

10. Dedoussis GVZ, Panagiotakos DB, Pitsavos C, Chrysohoou C, Skoumas J, Choumerianou $D$, et al. An association between the methylenetetrahydrofolate reductase (MTHFR) C677T mutation and inflammation markers related to cardiovascular disease. Int J Cardiol. 2005;100:409-14.

11. Morita H, Taguchi J, Kurihara H, Kitaoka M, Kaneda H, Kurihara Y, et al. Gene Polymorphism of 5, 10-methylenetetrahydrofolate reductase as a coronary risk factor. J Cardiol. 1997;29:309-15.

12. Tsai MY, Welge BG, Hanson NQ, Bignell MK, Vessey J, Schwichtenberg K, et al. Genetic causes of mild hyperhomocysteinemia in patients with premature occlusive coronary artery diseases. Atherosclerosis. 1999;143:163-70.

13. Bova I, Chapman J, Sylantiev C, Korczyn AD, Bornstein NM. The A677V methylenetetrahydrofolate reductase gene polymorphism and carotid atherosclerosis. Stroke. 1999:30:2180-2.

14. Jee SH, Song KS, Shim WH, Kim HK, Suh I, Park JY, et al. Major gene evidence after MTHFR-segregation analysis of serum homocysteine in families of patients undergoing coronary arteriography. Hum Genet. 2002;111:128-35.

15. Kolling K, Ndrepepa G, Koch W, Braun S, Mehilli J, Schomig A, et al. Methylenetetrahydrofolate reductase gene C677T and A1298C polymorphisms, plasma homocysteine, folate, and vitamin B12 levels and the extent of coronary artery disease. Am J Cardiol. 2004;93:1201-6.

16. Gueant-Rodriguez RM, Juilliere Y, Candito M, Adjalla CE, Gibelin P, Herbeth $B$, et al. Association of MTRRA66G polymorphism (but not of MTHFR C677T and A1298C, MTRA2756G, TCN C776G) with homocysteine and coronary disease in the French population. Thromb Haemost. 2005;94:510-5

17. Muniz MTC, Siqueira ERF, Fonseca RA, D'Almeida V, Hotta JK, Santos JE, et al. Avaliação da relação entre o polimorfismo C677T no gene para MTHFR e a concentração plamática de homocisteína na doença arterial coronariana. Arq Bras Endocrinol Metab. 2006;50:1059-65.

18. Arruda VR, Siqueira LH, Goncalves MS, Von Zuben PM, Soares MC, Menezes $\mathrm{R}$, et al. Prevalence of the mutation C677--> T in the methylenetetrahydrofolate reductase gene among distinct ethnic groups in Brazil. Am J Med. 1998;78:332-5

19. Abdel-Rahman SZ, Nouraldeen AM, Ahmed AE. Molecular interaction of 2,3-[14C]- acrylonitrile with DNA in gastric tissues of rat. J Biochem Toxicol. 1994;9:12128.

20. Ranjith N, Pegoraro RJ, Rom L. Risk factors and methylenetetrahydrofolate reductase gene polymorphisms in a young South African Indianbased population with acute myocardial infarction. Cardiovasc J South Afr. 2003; 14:127-32.

21. Welch GN, Loscalzo J. Homocysteine and atherothrombosis. N Engl J Med. 1998;338:1042-50.

22. Wang G, Woo CW, Sung FL, Siow YL, O K. Increased monocyte adhesion to aortic endothelium in rats with hyperhomocysteinemia: role of chemokine and adhesion molecules. Arterioscler Thromb Vasc Biol. 2002;22:1777-83.

23. Silverman MD, Tumuluri RJ, Davis M, Lopez G, Rosenbaum JT, Lelkes PI. Homocysteine upregulates vascular cell adhesion molecule-1 expression in 
cultured human aortic endothelial cells and enhances monocyte adhesion. Arterioscler Thromb Vasc Biol. 2002;22:587-92.

24. Kebert CB, Eichner JE, Moore WE, Schechter E, Yaoi T, Vogel S, et al. Relationship of the 1793G-A and 677C-T polymorphisms of the 5,10-methylenetetrahydrofolate reductase gene to coronary artery disease. Dis Markers 2006;22:293-301.

25. Bennouar N, Allami A, Azeddoug $H$, Bendris A, Laraqui A, El Jaffali A, et al. Thermolabile methylenetetrahydrofolate reductase $\mathrm{C} 677 \mathrm{~T}$ polymorphism and homocysteine are risk factors for coronary artery disease in moroccan population. J Biomed Biotechnol. 2007;2007:806-87.

26. Guerzoni AR, Pavarino-Bertelli EC, Godoy MF, Graça CR, Biselli PM, Souza DR, et al. Methylenetetrahydrofolate reductase gene polymorphism and its association with coronary artery disease. São Paulo Med J. $2007 ; 125: 4-8$.
27. Abu-Amero KK, Wyngaard CA, Dzimiri N. Prevalence and role of methylenetetrahydrofolate reductase $677 \mathrm{C} \otimes$ T and $1298 \mathrm{~A} \circledast \mathrm{C}$ polymorphisms in coronary artery disease in arabs. Arch Pathol Lab Med. 2003;127:1349-52.

28. Meisel C, Cascorbi I, Gerloff T, StangI V, Laule M, Müller JM. Identification of six methylenetetrahydrofolate reductase (MTHFR) genotypes resulting from common polymorphisms: impact on plasma homocysteine levels and development of coronary artery disease. Atherosclerosis. 2001;15.

Artigo recebido: 13/11/07

Aceito para publicação: 02/11/08 